\section{Validade das informações das declarações de nascidos vivos com base em estudo de caso-controle}

\author{
Validation of birth certificates based \\ on data from a case-control study
}

\author{
Márcia Furquim de Almeida 1 \\ Gizelton Pereira Alencar 1 \\ Ivan França Jr. ${ }^{1}$ \\ Hillegonda Maria Dutilh Novaes 2 \\ Arnaldo Augusto Franco de Siqueira 1 \\ Daniela Schoeps 1 \\ Oona Campbell 3 \\ Laura Cunha Rodrigues 3
}

\footnotetext{
1 Faculdade de Saúde Pública, Universidade de São Paulo, São Paulo, Brasil.

2 Faculdade de Medicina,

Universidade de São Paulo, São Paulo, Brasil.

3 Epidemiology Unit, London School of Hygiene and Tropical Medicine, London, UK.

Correspondência M. F. Almeida Departamento de Epidemiologia, Faculdade de Saúde Pública, Universidade de São Paulo. Av. Dr. Arnaldo 715, São Paulo, SP 01246-904, Brasil. marfural@usp.br
}

\begin{abstract}
The information recorded on birth certificates was validated with data from a perinatal mortality case-control study, obtained from home interviews of mothers and hospital records for cases (early neonatal deaths) and controls. Sensitivity, specificity, and concordance were calculated for all variables and their estimated and real prevalence. The completeness of birth certificates was lowest for mother's parity and presence of congenital anomalies (records without information range from $23 \%$ to $31 \%$ for cases and controls). Birth certificates correctly identified low birth weight and type of delivery for cases and controls. Birth certificates showed high sensitivity and specificity to detect preterm births within cases. The number of preterm births was underestimated at $30.8 \%$ of the controls and $2.9 \%$ of the cases. Low maternal education was two times greater on birth certificates than in the mother's interview, for cases and controls. Completeness of birth certificates was higher in controls, but data quality was better in cases.
\end{abstract}

Birth Certificate; Reliability and Validity; Records

\section{Introdução}

O SINASC (Sistema de Informações sobre Nascidos Vivos) foi implantado em 1990 e apresenta cobertura crescente de eventos no país. A razão entre o número de nascidos vivos captados pelo sistema e aquele obtido por meio de estimativas com base em dados censitários e de pesquisas amostrais pelo Instituto Brasileiro de Geografia e Estatística (IBGE) situava-se em torno de $90 \%$, entre 1999 e 20011 . As informações do SINASC têm sido utilizadas de forma intensiva e com diferentes finalidades: obtenção de indicadores de saúde, estudos epidemiológicos e atividades de vigilância à saúde.

Estudos nacionais de avaliação da qualidade das informações mostram que o SINASC apresenta boa completude de dados $2,3,4,5$. No entanto, ainda que condição necessária, ela não é suficiente para atender as crescentes exigências relativas à qualidade dos sistemas de informação em saúde, como pode-se observar pela evolução nos estudos de avaliação dos sistemas de informações perinatais nos Estados Unidos 6. Inicialmente, estes estudos destinavam-se apenas à avaliação da completude dos dados, evoluindo para avaliação de sua confiabilidade por meio da concordância dos registros com outras fontes de dados 7,8.

Alguns estudos avaliam a confiabilidade das medidas por meio do índice kappa 9,10,11, que mede o quanto se está distante da aleatorieda- 
de e da concordância perfeita. Nesses estudos, não há definição da fonte de referência a ser empregada.

Os estudos de validação de sistemas de informações que adotam uma fonte de dados como padrão-ouro permitem obter medidas de sensibilidade, especificidade e, quando apropriado, valores preditivos. Este tipo de estudo foi realizado por Reichman \& Hade 12, comparando os dados dos certificados de nascimento e a base de dados proveniente da atenção prénatal e ao parto do programa Health-Start Maternity Services Summary Data. A definição de antemão da fonte de dados que será considerada como referência permite a obtenção de medidas de mais fácil interpretação para a validação da informação como, por exemplo, sensibilidade, especificidade e valores preditivos, e a comparação entre as prevalências real (pesquisa) e a estimada que é obtida nos sistemas de informação.

As informações dos prontuários hospitalares são freqüentemente utilizadas como referência para a avaliação dos sistemas de informação em saúde tanto em estudos nacionais 7 como internacionais 8,12,13. O estudo de MelloJorge et al. 7 , realizado em cinco municípios paulistas, mostrou que algumas variáveis, como por exemplo o peso ao nascer, apresentavam excelente completude e elevado grau de concordância com os dados registrados nos prontuários médicos. Também têm sido utilizadas informações obtidas em entrevistas individuais para comparação dos dados do sistema de informação sobre nascidos vivos 9,10.

A escolha da fonte de dados que será considerada como padrão-ouro depende da variável em estudo. Para algumas variáveis, como aquelas que formam o bloco de características das mães na declaração de nascido vivo, as informações obtidas diretamente com as mães, por meio de entrevistas, podem ser mais adequadas, pois evitam possíveis erros de registro ou de transcrição de dados para prontuários médicos. Como exemplo, a informação sobre o número de consultas de pré-natal realizadas nem sempre é registrada no prontuário hospitalar, assim a informação obtida diretamente com a mãe pode ser mais fidedigna. Já para outras variáveis, a fonte mais adequada para ser considerada como padrão-ouro é o prontuário médico como, por exemplo, o peso ao nascer. Esta informação é obtida diretamente pelos serviços de saúde e posteriormente fornecida para a mãe; ao se utilizar o prontuário médico estar-se-á evitando possíveis erros de memória da mãe.

Vários estudos de validação dos sistemas de informação de nascidos vivos tiveram origem em estudos de tipo caso-controle de base populacional 8,9. Uma vantagem deste tipo de estudo é a possibilidade de identificar se há diferença na qualidade do registro de dados nos sobreviventes e nas crianças que morreram, como foi identificado por Piper et al. 8 .

A pesquisa Mortalidade Perinatal na Região Sul do Município de São Paulo: Um Estudo Caso-controle de Base Populacional, ao realizar entrevistas domiciliares com as mães e ao empregar um protocolo para obter dados do prontuário hospitalar, criou a possibilidade de utilizar estas duas fontes de dados para avaliar as informações do SINASC, objetivo deste trabalho.

\section{Material e métodos}

Para obter a amostra utilizada no estudo foi realizada "linkage" dos bancos de dados referentes aos nascidos vivos de mães residentes na região sul do Município de São Paulo, cujo parto ocorreu no próprio município ou em municípios da região metropolitana de São Paulo, no período de 1o de agosto de 2000 a 31 de janeiro de 2001, e o banco de dados de óbitos neonatais (menores de 28 dias) no período de 1 o de agosto de 2000 a 26 de fevereiro de 2001 , definindo como casos os óbitos neonatais precoces e como controles uma amostra aleatória dos sobreviventes. Após a identificação dos indivíduos da amostra obteve-se cópia das declarações de nascido vivo (DN) na Fundação Sistema Estadual de Análise de Dados (Fundação SEADE).

Foram utilizadas informações obtidas em entrevistas domiciliares com as mães, e foi aplicado protocolo padronizado para obter informações nos prontuários hospitalares das mães e dos recém-nascidos, para 146 casos (óbitos neonatais precoces) e 313 controles.

Para avaliar o grau de preenchimento das DN considerou-se como ausentes os "brancos" (não preenchimento) e os ignorados. Na pesquisa considerou-se como ausência de informação a não obtenção da informação nas entrevistas domiciliares ou quando esta se mostrou inconsistente com as demais informações obtidas. No protocolo para coleta de dados nos prontuários médicos havia o quesito para identificar a ausência de registro no prontuário médico.

Para avaliar possíveis diferenças no preenchimento das DN de casos e controles utilizouse o qui-quadrado de Pearson ou, quando necessário, o teste exato de Fisher considerandose um valor de $\mathrm{p}<0,05$

Considerou-se como padrão-ouro as informações obtidas nas entrevistas domiciliares pa- 
ra as variáveis: estado civil, pré-natal, paridade, idade da mãe e tipo de parto. Os prontuários hospitalares foram considerados como padrão-ouro para as variáveis: duração da gestação, peso ao nascer e presença de anomalias congênitas.

As informações obtidas nas entrevistas e nos prontuários foram inicialmente trabalhadas de modo a se obter as mesmas categorias das variáveis existentes na DN.

A idade materna foi reagrupada em menores de 20 anos (mães adolescentes) e 20 anos e mais. Na entrevista domiciliar obteve-se a data de nascimento da mãe e a idade foi obtida subtraindo-se da data do parto. O número total de filhos anteriores (vivos e mortos) passou a ser denominado de paridade. O tipo de parto foi agrupado em vaginal (normal e fórceps) e cesáreo. O estado marital da mãe foi composto de modo a identificar se a mãe, no momento do parto, tinha ou não companheiro e, assim, foram agregadas as categorias viúvas e separadas judicialmente às solteiras e as unidas em união consensual às casadas. A assistência pré-natal foi considerada como fez ou não fez pré-natal, o número de consultas de pré-natal foi considerado como inadequado quando havia menos de sete consultas. Foram consideradas como sem informação sobre a presença de anomalias congênitas as DN que não apresentavam o campo 34 com anotação no quesito sim e não.

$\mathrm{O}$ peso ao nascer foi dicotomizado em menos de $2.500 \mathrm{~g}$ (baixo peso) e $2.500 \mathrm{~g}$ e mais. A variável duração da gestação foi agrupada em pré-termo (até 37 semanas) e não pré-termo (37 semanas e mais). A variável duração da gestação foi obtida por meio do seguinte algoritmo: inicialmente essa informação foi obtida nos prontuários hospitalares do recém-nascido, considerando a seguinte hierarquia: (1) informação sobre a data da realização de ultrasonografia (por se considerar esta medida mais precisa); (2) idade gestacional registrada em semanas, obtida por diferentes métodos de avaliação clínica (Dubowitz, Ballard e Capurro); (3) a informação da data da última menstruação registrada no prontuário obstétrico; e (4) a data da última menstruação obtida na entrevista domiciliar. Como medida de confiabilidade, calculou-se o grau de concordância como a proporção de concordantes das duas fontes considerando para casos e controles. A concordância foi considerada como elevada quando seu valor era igual ou maior que $90 \%$, moderada quando se situava entre $70 \%$ e $89 \%$, e baixa quando era menor que $70 \% 7$. As medidas utilizadas para avaliar a validade foram: sensibilidade, especificidade e a razão entre as pre- valências real e estimada. A sensibilidade foi definida como a proporção de indivíduos que apresentavam dada condição na fonte definida como padrão-ouro que foi corretamente identificada nas DN. A especificidade foi definida como a proporção de indivíduos que não apresentavam dada condição na fonte definida como padrão-ouro e que foi corretamente identificada nas DN. Considerou-se a prevalência real como aquela resultante da pesquisa e a estimada aquela obtida no SINASC, segundo Pereira 14.

Adotou-se o critério proposto por Piper et al. 8 , que definiu sensibilidade elevada quando esta apresenta valor acima de $90 \%$, como moderada entre $70-90 \%$ e baixa menor que $70 \%$. Não foram avaliadas a sensibilidade e a especificidade das variáveis que apresentaram proporção de não-preenchimento igual ou superior a $30 \%$.

\section{Resultados}

As variáveis descritivas das características da mãe apresentaram deficiência de preenchimento na DN: a paridade apresentou pior resultado, estando ausente em um quarto dos nascimentos estudados. As demais variáveis apresentaram ausência de até $10 \%$, porém sua ausência é significativamente maior nos casos que nos controles (Tabela 1).

As variáveis presença e adequação do número de consultas de pré-natal também apresentaram significativas diferenças no preenchimento de casos e controles, ao passo que o tipo de parto teve a menor ausência de informação e não mostrou diferença significativa entre casos e controles (Tabela 1).

Com relação às características do recémnascido, a variável presença de anomalia congênita esteve ausente em cerca de $30 \%$ de casos e controles. A duração da gestação e o peso ao nascer apresentaram pequena proporção de ausência de informação, porém estas foram significativamente mais elevadas nos casos do que nos controles (Tabela 1).

As Tabelas 2 e 3 contemplam a avaliação das informações registradas na DN para casos e controles, respectivamente, por meio das medidas de confiabilidade: grau de concordância das duas fontes de dados; e de validade por meio da sensibilidade, especificidade, prevalência real (pesquisa) e a estimada (DN), e relação entre as prevalências estimada e real. Não foram calculadas as medidas de confiabilidade e de validade para as variáveis paridade e presença de anomalias congênitas (ausência de informação de cerca de 30\%). 
Proporção de ausência de informação nas declarações de nascido vivo (DN) e pesquisa.

Região sul do Município de São Paulo, Brasil, 2000/2001.

\begin{tabular}{|c|c|c|c|c|c|c|c|c|c|c|c|c|c|c|c|}
\hline \multirow[t]{3}{*}{ Variáveis } & \multicolumn{4}{|c|}{ Casos (146) } & \multicolumn{4}{|c|}{ Controles (313) } & \multicolumn{4}{|c|}{ Total (459) } & \multirow{2}{*}{\multicolumn{3}{|c|}{$\begin{array}{l}\text { Diferença entre } \\
\text { casos e controles }\end{array}$}} \\
\hline & \multicolumn{2}{|c|}{ DN } & \multicolumn{2}{|c|}{ Pesquisa } & \multicolumn{2}{|c|}{ DN } & \multicolumn{2}{|c|}{ Pesquisa } & \multicolumn{2}{|c|}{ DN } & \multicolumn{2}{|c|}{ Pesquisa } & & & \\
\hline & $n$ & $\%$ & $\mathrm{n}$ & $\%$ & $n$ & $\%$ & $\mathrm{n}$ & $\%$ & $\mathrm{n}$ & $\%$ & $n$ & $\%$ & $\chi^{2}$ & gl & $p$ \\
\hline \multicolumn{16}{|l|}{ Características da mãe } \\
\hline Estado marital & 17 & 11,64 & 0 & 0,00 & 11 & 3,51 & 0 & 0,00 & 28 & 6,10 & 0 & 0,00 & * & & $<0,001$ \\
\hline Idade & 8 & 5,48 & 0 & 0,00 & 0 & 0,00 & 0 & 0,00 & 8 & 1,74 & 0 & 0,00 & 1,38 & 1 & 0,24 \\
\hline Paridade & 41 & 28,08 & 0 & 0,00 & 72 & 23,00 & 0 & 0,00 & 113 & 24,62 & 0 & 0,00 & 4,33 & 1 & 0,037 \\
\hline Anos de estudo & 22 & 15,07 & 0 & 0,00 & 15 & 4,79 & 0 & 0,00 & 37 & 8,06 & 0 & 0,00 & 11,49 & 1 & $<0,001$ \\
\hline \multicolumn{16}{|l|}{ Gestação e parto } \\
\hline Tipo de parto & 5 & 3,42 & 0 & 0,00 & 3 & 0,96 & 0 & 0,00 & 8 & 1,74 & 0 & 0,00 & * & & 0,117 \\
\hline $\begin{array}{l}\text { Presença de assistência } \\
\text { pré-natal }\end{array}$ & 30 & 20,55 & 0 & 0,00 & 27 & 8,63 & 0 & 0,00 & 57 & 12,42 & 0 & 0,00 & 13,01 & 1 & $<0,001$ \\
\hline $\begin{array}{l}\text { Número de consultas } \\
\text { de pré-natal }\end{array}$ & 30 & 20,55 & 0 & 0,00 & 27 & 8,63 & 10 & 3,19 & 57 & 12,42 & 10 & 2,18 & 13,01 & 1 & $<0,001$ \\
\hline \multicolumn{16}{|l|}{ Recém-nascido } \\
\hline Duração da gestação & 9 & 6,16 & 5 & 3,42 & 3 & 0,96 & 0 & 0,00 & 12 & 2,61 & 5 & 1,09 & * & & 0,001 \\
\hline Peso & 5 & 3,42 & 1 & 0,68 & 0 & 0,00 & 0 & 0,00 & 5 & 1,09 & 1 & 0,22 & * & & 0,001 \\
\hline $\begin{array}{l}\text { Presença de anomalia } \\
\text { congênita }\end{array}$ & 45 & 30,82 & 0 & 0,00 & 91 & 29,07 & 0 & 0,00 & 136 & 29,63 & 0 & 0,00 & 0,15 & 1 & 0,702 \\
\hline
\end{tabular}

* Teste exato de Fisher.

A concordância da idade registrada na DN com a data de nascimento informada pela mãe pode ser considerada como alta (cerca de $95 \%$ para casos) e moderada (cerca de $85 \%$ para controles). Quanto à identificação de mães adolescentes, verificou-se existir sensibilidade e especificidade elevadas, $90,2 \%$ e $98 \%$, respectivamente. A diferença entre as prevalências estimada e real é pequena para os casos, porém, a DN subestima em cerca de $46 \%$ a presença de mães adolescentes nos controles (Tabelas 2 e 3).

A concordância entre as mulheres que informaram ter menos de três anos de estudo e a informação da DN situou-se em torno de $62 \%$, que pode ser considerada como baixa para casos e controles. Para captar a presença de baixa escolaridade materna, a DN demonstrou ter elevada sensibilidade (acima de $90 \%$ ), no entanto, mostrou baixa especificidade, tanto para casos como para controles $(60 \%)$ indicando existir falsos positivos. O SINASC superestima em cerca de duas vezes a presença de baixa escolaridade tanto para casos como para controles (Tabelas 2 e 3 ).

A concordância entre a informação fornecida pela mãe sobre seu estado marital e aquela registrada na DN, situou-se em torno de $71 \%$ e $78 \%$, respectivamente, para casos e controles. A condição de mãe sem companheiro apresentou moderada sensibilidade, em torno de $71 \%$ nos casos e $75 \%$ nos controles. A DN superestima igualmente a prevalência de mães sem companheiro em $75 \%$ para os casos e controles (Tabelas 2 e 3 ).

A concordância entre a pesquisa e a $\mathrm{DN}$ com relação às mães não terem recebido assistência pré-natal é elevada (acima de $90 \%$ ) tanto para casos como para controles. Com relação à identificação das mães que não fizeram pré-natal, a sensibilidade pode ser considerada como moderada para casos $(73,3 \%)$ e controles $(75 \%)$. Verificou-se elevada especificidade para identificação de mães que não realizam pré-natal. Com relação ao número adequado de consultas de pré-natal, verifica-se uma redução da concordância das informações das duas fontes, tanto para os casos (63\%) como para os controles (68\%). Observa-se, ainda, que a DN não é um instrumento sensível para identificar as mães com número insuficiente de consultas. Nos controles, a sensibilidade é inferior a $24 \%$ e nos casos, é de $54 \%$. Como conseqüência, a DN subestima a freqüência de mulheres com número de consultas inadequadas para os casos $(40 \%)$ e controles $(72 \%)$.

As DN podem ser consideradas como excelente fonte de identificação de recém-nascidos de baixo peso ao nascer e do tipo de parto realizado, pois apresentaram o grau de concordância mais elevado entre os dados registrados 
Informações registradas nas declarações de nascido vivo (DN) e obtidas em inquérito domiciliar e prontuários médicos nos 146 casos*. Região sul do Município de São Paulo, Brasil, 2000/2001.

\begin{tabular}{|c|c|c|c|c|c|c|c|c|c|}
\hline Variáveis na DN & $\begin{array}{c}\text { Categor } \\
\text { i. }\end{array}$ & $\begin{array}{l}\text { ii. } \\
\text { ii }\end{array}$ & Total & $\begin{array}{l}\text { Concor- } \\
\text { dância }\end{array}$ & $\begin{array}{l}\text { Sensibi- } \\
\text { lidade }\end{array}$ & $\begin{array}{l}\text { Especifi- } \\
\text { cidade }\end{array}$ & $\begin{array}{l}\text { Prevalência } \\
\text { real }\end{array}$ & $\begin{array}{l}\text { Prevalência } \\
\text { estimada }\end{array}$ & $\begin{array}{c}\text { Prevalência } \\
\text { estimada/ } \\
\text { Real (\%) }\end{array}$ \\
\hline i. Mãe sem companheiro & 20 & 29 & 49 & 71,32 & 71,43 & 71,29 & 21,71 & 37,98 & 75,00 \\
\hline ii. Mãe com companheiro & 8 & 72 & 80 & & & & & & \\
\hline Total & 28 & 101 & 129 & & & & & & \\
\hline i. Idade da mãe até 20 anos & 37 & 2 & 39 & 95,65 & 90,24 & 97,94 & 29,71 & 28,26 & $-4,88$ \\
\hline $\begin{array}{l}\text { ii. Idade da mãe } 20 \text { anos } \\
\text { e mais }\end{array}$ & 4 & 95 & 99 & & & & & & \\
\hline Total & 41 & 97 & 138 & & & & & & \\
\hline $\begin{array}{l}\text { i. Mãe com até } 3 \text { anos } \\
\text { de estudo }\end{array}$ & 14 & 47 & 61 & 60,66 & 93,33 & 56,07 & 12,30 & 50,00 & 306,67 \\
\hline $\begin{array}{l}\text { ii. Mãe com } 4 \text { anos e mais } \\
\text { de estudo }\end{array}$ & 1 & 60 & 61 & & & & & & \\
\hline Total & 15 & 107 & 122 & & & & & & \\
\hline i. Tipo de parto vaginal & 90 & 0 & 90 & 100,00 & 100,00 & 100,00 & 63,83 & 63,83 & 0,00 \\
\hline ii. Tipo de parto cesáreo & 0 & 51 & 51 & & & & & & \\
\hline Total & 90 & 51 & 141 & & & & & & \\
\hline i. Mãe não fez pré-natal & 11 & 3 & 14 & 93,91 & 73,33 & 97,00 & 13,04 & 12,17 & $-6,67$ \\
\hline ii. Mãe fez pré-natal & 4 & 97 & 101 & & & & & & \\
\hline Total & 15 & 100 & 115 & & & & & & \\
\hline $\begin{array}{l}\text { i. Número inadequado } \\
\text { de consultas de pré-natal** }\end{array}$ & 44 & 5 & 49 & 62,93 & 53,66 & 85,29 & 70,69 & 42,24 & $-40,24$ \\
\hline $\begin{array}{l}\text { ii. Número adequado de } \\
\text { consultas de pré-natal }\end{array}$ & 38 & 29 & 67 & & & & & & \\
\hline i. Baixo peso ao nascer $r^{\star \star \star}$ & 112 & 0 & 112 & 98,57 & 98,25 & 100,00 & 81,43 & 80,00 & $-1,75$ \\
\hline ii. Não-baixo peso ao nascer & 2 & 26 & 28 & & & & & & \\
\hline Total & 114 & 26 & 140 & & & & & & \\
\hline i. Pré-termo*** & 96 & 4 & 100 & 91,73 & 93,20 & 86,67 & 77,44 & 75,19 & $-2,91$ \\
\hline ii. Não pré-termo & 7 & 26 & 33 & & & & & & \\
\hline Total & 103 & 30 & 133 & & & & & & \\
\hline
\end{tabular}

* Excluiu-se ignorados da DN e da pesquisa;

** Excluiu-se mães sem pré-natal;

*** Padrão-ouro é o prontuário hospitalar.

na DN e os prontuários hospitalares (próximo de $100 \%$ ), em casos e controles. Verificou-se também existir elevadas sensibilidade e especificidade para casos e controles. Como conseqüência, as prevalências real e estimada apresentam valores próximos. Contudo, observa-se que a DN subestima o baixo peso ao nascer em $1,9 \%$ dos casos e superestima a presença desta condição em $8 \%$ nos controles (Tabelas 2 e 3 ).

A concordância entre as informações da DN e da pesquisa para os nascimentos de pré-ter- mo foi elevada para casos $(91,7 \%)$ e controles $(96,1 \%)$. Nos casos verificou-se que a sensibilidade $(93,2 \%)$ e a especificidade $(86,7 \%)$ apresentaram resultados próximos ao parâmetro considerado como elevado (90\%). Há uma pequena diferença entre a prevalência real e a estimada $(2,9 \%)$, indicando que a DN identifica adequadamente as gestações de pré-termo entre os nascimentos que resultaram em óbito neonatal precoce. No entanto, nos controles há baixa sensibilidade $(61,5 \%)$ para identificar os 
Tabela 3

Informações registradas nas declarações de nascido vivo (DN) e obtidas em inquérito domiciliar e prontuários médicos nos 313 controles*. Região sul do Município de São Paulo, Brasil, 2000/2001.

\begin{tabular}{|c|c|c|c|c|c|c|c|c|c|}
\hline Variáveis na DN & $\begin{array}{c}\text { Categor } \\
\text { i. }\end{array}$ & $\begin{array}{l}\text { esquisa } \\
\text { ii. }\end{array}$ & Total & $\begin{array}{l}\text { Concor- } \\
\text { dância }\end{array}$ & $\begin{array}{l}\text { Sensibi- } \\
\text { lidade }\end{array}$ & $\begin{array}{l}\text { Especifi- } \\
\text { cidade }\end{array}$ & $\begin{array}{l}\text { Prevalência } \\
\text { real }\end{array}$ & $\begin{array}{l}\text { Prevalência } \\
\text { estimada }\end{array}$ & $\begin{array}{c}\text { Prevalência } \\
\text { estimada/ } \\
\text { Real (\%) }\end{array}$ \\
\hline i. Mãe sem companheiro & 39 & 52 & 91 & 78,48 & 75,00 & 79,20 & 17,22 & 30,13 & 75,00 \\
\hline ii. Mãe com companheiro & 13 & 198 & 211 & & & & & & \\
\hline Total & 52 & 250 & 302 & & & & & & \\
\hline i. Idade da mãe até 20 anos & 53 & 1 & 54 & 84,66 & 53,00 & 99,53 & 31,95 & 17,25 & $-46,00$ \\
\hline $\begin{array}{l}\text { ii. Idade da mãe } 20 \text { anos } \\
\text { e mais }\end{array}$ & 47 & 212 & 259 & & & & & & \\
\hline Total & 100 & 213 & 313 & & & & & & \\
\hline $\begin{array}{l}\text { i. Mãe com até } 3 \text { anos } \\
\text { de estudo }\end{array}$ & 29 & 109 & 138 & 62,42 & 90,63 & 59,02 & 10,74 & 46,31 & 331,25 \\
\hline $\begin{array}{l}\text { ii. Mãe com } 4 \text { anos e mais } \\
\text { de estudo }\end{array}$ & 3 & 157 & 160 & & & & & & \\
\hline Total & 32 & 266 & 298 & & & & & & \\
\hline i. Tipo de parto vaginal & 163 & 3 & 166 & 98,71 & 99,39 & 97,95 & 52,90 & 53,55 & 1,22 \\
\hline ii. Tipo de parto cesáreo & 1 & 143 & 144 & & & & & & \\
\hline Total & 164 & 146 & 310 & & & & & & \\
\hline i. Mãe não fez pré-natal & 3 & 3 & 6 & 98,60 & 75,00 & 98,94 & 1,40 & 2,10 & 50,00 \\
\hline ii. Mãe fez pré-natal & 1 & 279 & 280 & & & & & & \\
\hline Total & 4 & 282 & 286 & & & & & & \\
\hline $\begin{array}{l}\text { i. Número inadequado de } \\
\text { consultas de pré-natal** }\end{array}$ & 26 & 5 & 31 & 67,75 & 23,64 & 96,99 & 39,86 & 11,23 & $-71,82$ \\
\hline $\begin{array}{l}\text { ii. Número adequado de } \\
\text { consultas de pré-natal }\end{array}$ & 84 & 161 & 245 & & & & & & \\
\hline Total & 110 & 166 & 276 & & & & & & \\
\hline i. Baixo peso ao nascer ${ }^{\star \star \star}$ & 25 & 2 & 27 & 99,36 & 100,00 & 99,31 & 7,99 & 8,63 & 8,00 \\
\hline ii. Não-baixo peso ao nascer & 0 & 286 & 286 & & & & & & \\
\hline Total & 25 & 288 & 313 & & & & & & \\
\hline 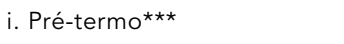 & 16 & 2 & 18 & 96,13 & 61,54 & 99,30 & 8,39 & 5,81 & $-30,77$ \\
\hline ii. Não pré-termo & 10 & 282 & 292 & & & & & & \\
\hline Total & 26 & 284 & 310 & & & & & & \\
\hline
\end{tabular}

* Excluiu-se ignorados da DN e da pesquisa;

** Excluiu-se mães sem pré-natal.

*** Padrão-ouro é o prontuário hospitalar.

nascimentos de pré-termo, e como conseqüência a diferença entre a prevalência real $(8,4 \%)$ e a estimada pela DN $(5,8 \%)$, indicando que o SINASC subestima em $30,8 \%$ a presença desta condição (Tabelas 2 e 3).

\section{Discussão}

Os sobreviventes apresentaram maior completude de registro de dados que os recém-nasci- dos que morreram antes de completar sete dias para todas as variáveis estudadas. As diferenças encontradas no grau de preenchimento de informações para casos e controles foram significativas para quase todas as variáveis estudadas; resultado semelhante foi encontrado por Piper et al. 8, em estudo de caso-controle realizado no Tennessee, Estados Unidos. As variáveis paridade e presença de anomalias congênitas apresentaram elevada ausência de informação e não houve diferença significativa 
entre casos e controles, indicando que a ausência de registro de informações na DN distribuise igualmente entre os sobreviventes e os recém-nascidos que morreram.

Com relação à paridade materna, Mello-Jorge et al. 7, em estudo de 1992, já haviam identificado a precariedade de registro desses dados na DN. Este fato pode ter origem na dificuldade de se transcrever os dados registrados nos prontuários médicos para a DN; alguns prontuários registram o número de gestações anteriores enquanto outros registram o número de partos anteriores. Além disso, alguns prontuários identificam o número de abortos espontâneos anteriores e outros não. Para completar a dificuldade de coleta de dados a DN, nesse campo, emprega a denominação de nascidos mortos anteriores, o que pode trazer dúvidas para os serviços de saúde de qual informação deve ser registrada, apenas os nascimentos com mais de $1.000 \mathrm{~g}$ e/ ou de 28 semanas e mais, ou todas as perdas fetais anteriores incluindo aqui os abortos espontâneos.

A variável presença de anomalia congênita do recém-nascido foi introduzida apenas na última versão da DN, o que pode explicar, em parte, sua elevada ausência de registro. Watkins et al. 15, em estudo realizado na Georgia, Estados Unidos, encontraram menor identificação nos certificados de nascimento que nos prontuários hospitalares para os defeitos congênitos, sendo que o registro era mais freqüente para as anomalias congênitas mais facilmente identificáveis (alterações externas ou internas graves).

As variáveis sócio-econômicas presentes na DN situação marital e anos de estudo das mães apresentaram boa completude. Com relação ao estado marital, verifica-se que as informações do SINASC superestimam igualmente a presença de mães sem companheiro para casos e controles, possivelmente este fato se deva ao registro como mulheres solteiras para todas aquelas que não tenham união legal, ou seja, a informação registrada refere-se de fato ao estado civil.

Com relação à variável número de anos de estudo da mãe, a DN identifica corretamente as mães de baixa escolaridade, porém superestima em mais de duas vezes sua prevalência em casos e controles. Provavelmente, este fato se deve à forma como este dado é coletado nos hospitais. Cerca de $70 \%$ dos casos e controles deste estudo tiveram seu parto pago pelo SUS; é possível que os hospitais assumam tratar-se de população de baixa escolaridade e nem sequer perguntam diretamente às mães os anos de estudo que tenham completado. Esse pode ser um dos componentes desse resultado. Ou- tro componente derivado desse pode ser a pouca importância que os serviços de saúde dão à escolaridade materna e ao seu registro adequado. Esta hipótese se respalda no resultado observado na pesquisa no qual se verificou que a escolaridade materna se encontrava ausente nos prontuários hospitalares de $65,8 \%$ dos sobreviventes e 58,9\% dos óbitos neonatais precoces, que seria a fonte para a coleta de dados da DN. Note-se que esta variável também foi modificada na última versão da DN que entrou em vigor em 1998. Nas versões anteriores da DN a variável utilizada era grau de instrução das mães. O estudo já citado de Mello-Jorge et al. 7, mostrou que para essa variável foi possível comparar os registros da DN com os prontuários médicos em apenas $32,5 \%$ dos nascidos vivos estudados, devido à elevada ausência de registro da instrução materna nos prontuários hospitalares. Estudo em São Luís, Maranhão, Brasil, também mostrou existirem baixos valores do índice kappa $(0,30)$ para a escolaridade materna entre a DN e as informações obtidas em inquérito realizado com as mães 9 .

Estudos anteriores mostraram que a informação sobre a idade materna registrada na DN apresentou boa concordância com as informações registradas nos prontuários médicos 7; resultado semelhante foi obtido quando esta foi comparada com idade informada pelas mães em entrevistas ${ }^{9}$. Ao se considerar as mães em adolescentes (menores de vinte anos) e nãoadolescentes, o grau de concordância entre a DN e a idade da mãe (obtida a partir da data de nascimento) foi alta para casos e moderada para os controles. Um possível viés de registro de dados que pode estar presente é os serviços de saúde estarem arredondando para cima a idade materna 16. A DN identifica menos esta condição nos controles que nos casos, nos quais verificou-se elevada sensibilidade, enquanto foi moderada nos controles.

O SINASC mostrou ser uma razoável fonte de dados para identificar as mães que não realizaram pré-natal, há elevada concordância com as informações obtidas junto às mães, porém a sensibilidade é moderada para casos e controles, mostrando que parte das mães que não receberam atenção pré-natal durante a gestação não foram identificadas na DN, sendo que este fato é mais evidente nos controles (50\%) que nos casos $(6,7 \%)$. Contudo, o número de mães que não fizeram pré-natal é muito pequeno, gerando elevada diferença entre as prevalências real e estimada.

A avaliação sobre a adequação do pré-natal restringe-se ao número de consultas realizadas, considerando-se sete consultas ou mais 
como adequado. A concordância com a informação prestada pela mãe sobre o número de consultas foi moderada, sendo levemente superior nos controles $(67,8 \%)$ que nos casos $(62,9 \%)$. Piper et al. 8 verificaram que a concordância sobre o número exato de visitas de prénatal era maior entre os casos que nos controles, enquanto que neste estudo definiu-se o parâmetro de sete consultas ou mais. Possivelmente, este fato se deve à ausência de registro sobre o número de consultas de pré-natal nos prontuários hospitalares, a pesquisa identificou que essa informação encontrava-se mais ausente nos prontuários dos óbitos neonatais precoces $(74 \%)$ que nos dos controles $(60,1 \%)$. Silva et al. 9 e Theme Filha et al. 11 encontraram baixo índice kappa, mostrando que as informações obtidas no SINASC não eram confiáveis para avaliar o número de consultas de pré-natal. Clark et al. 13, em estudo realizado na Geórgia, Estados Unidos, verificaram que a concordância sobre o número de visitas de pré-natal também era muito reduzida entre os certificados de nascimento e os prontuários médicos das clínicas onde as mães haviam realizado pré-natal. McDermott et al. 10, comparando as informações obtidas diretamente com as mães e as do certificado de nascimento, e utilizando uma classificação de número de consultas de pré-natal ajustada por idade gestacional verificaram que a concordância entre estas fontes era maior para os dois extremos, ou seja, para as mulheres que não fizeram pré-natal e aquelas com pré-natal adequado, do que para aquelas com pré-natal inadequado. Há grandes dificuldades na obtenção dessa informação, tendo em vista a multiplicidade de entendimento sobre o que se considera consulta de pré-natal. Pode-se considerar como consulta de pré-natal toda e qualquer visita das gestantes aos serviços de saúde, como pode-se considerar apenas a consulta médica completa; nem sempre é fácil para mãe estabelecer estas diferenças e por conseguinte informar adequadamente os serviços de atenção ao parto. Aos possíveis erros de entendimento e relato adiciona-se potenciais erros de registro e transcrição para a DN. Estas dificuldades encontram-se presentes na avaliação desta variável no SINASC.

O tipo de parto apresentou concordância de $100 \%$ nos casos e pouco menor nos controles, tanto nos estudos realizados no país 7,9 como naqueles realizados nos Estados Unidos 8,12,17, em que se observa que esta variável apresentase como confiável nos sistemas de informação.

O SINASC mostrou-se como fonte válida e confiável para a identificação de recém-nascidos de baixo peso ao nascer, este resultado foi semelhante a outros estudos 7,9,12. Os resultados mostraram que as informações registradas também podem ser consideradas como acuradas (elevada sensibilidade e especificidade). No entanto, verifica-se que o SINASC subestima levemente a prevalência de baixo peso ao nascer nos casos, e nos controles se encontra levemente superestimada.

A informação presente na DN é confiável (elevada concordância) para a identificação de nascimentos de pré-termo, porém se encontra subestimada, esta distorção é menor nos casos que nos controles. Este resultado é consistente com o obtido por Silva et al. 9, que mostravam que o SINASC subestimava o número de nascimentos de pré-termo. O SINASC é a principal fonte de dados populacionais para obter essa informação. Entretanto, é possível que ao se categorizar a variável duração da gestação na $\mathrm{DN}$, pode-se contribuir para sua incorreta anotação.

Os casos (óbitos de menores de sete dias) representam um número muito pequeno do total de nascimentos vivos, dado que este é um evento raro, porém os controles foram obtidos com base em uma amostra aleatória dos sobreviventes. Desse modo, pode-se inferir que a qualidade das informações registradas do SINASC aproxima-se mais do perfil encontrado nos controles.

As diferenças encontradas na completude, confiabilidade e validade dos dados registrados no SINASC para casos e controles merecem uma investigação mais detalhada para identificar as razões destas diferenças. Outras variáveis que não foram consideradas neste estudo podem influenciar estes resultados, tais como o momento em que são registradas as informações nas declarações de nascido vivo (se estas são preenchidas logo após o parto ou são preenchidas tempo depois com base nos dados existentes nos prontuários médicos). Podem existir também diferenças em relação a quem efetua o registro de dados, como por exemplo, se é o pediatra, enfermeira ou o pessoal administrativo do serviço de arquivo médico. Os casos e controles estudados ocorreram em 62 hospitais da região metropolitana de São Paulo e seguramente existem diferenças de rotina de registro de dados nos hospitais, dependendo se estes são universitários, contratados pelo SUS e privados.

\section{Conclusões}

A falta de registro de informações sobre a paridade materna e a presença de anomalias congênitas foi de tal magnitude que impediu sua 
validação. Tendo em vista que o SINASC pode ser utilizado como importante instrumento de monitoramento populacional de anomalias congênitas, devem ser feitos esforços para garantir melhor captação de informação.

$\mathrm{Na}$ análise das variáveis selecionadas observou-se que a completude dos dados foi significativamente superior nos controles que nos casos. As informações sócio-econômicas (esta- do marital e anos de estudo das mães) apresentaram menor validade que as demais, tanto nos casos como nos controles. O SINASC é uma excelente fonte de informação para identificar nascimentos de baixo peso ao nascer. Quanto à identificação de nascimentos de pré-termo esta é de melhor qualidade nos casos que nos controles.

\section{Resumo}

A pesquisa Mortalidade Perinatal na Região Sul do Município de São Paulo: Um Estudo Caso-controle de Base Populacional possibilitou a comparação das informações das declarações de nascido vivo (DN) com as obtidas em entrevistas com as mães e o registro nos prontuários hospitalares, e permitiu avaliar diferenças de registro nos nascimentos que resultaram em óbitos neonatais precoces (casos) e sobreviventes (controles). Para avaliar a confiabilidade e validade das informações das DN foram utilizadas medidas de concordância, sensibilidade, especificidade, prevalências real e estimada. A paridade materna e presença de anomalias congênitas mostraram elevada ausência de registro. O peso ao nascer e o tipo de parto são bem identificadas pela DN, em casos e controles. A DN mostrou alta sensibilidade e especificidade para captar os nascimentos de pré-termo nos casos, contudo, subestima o número de nascimentos de pré-termo: $30,8 \%$ nos controles e 2,9\% nos casos. O SINASC superestima em cerca de duas vezes a presença de nascimentos de mães de baixa escolaridade. Verificou-se que as DNs dos controles têm informações mais completas, porém a informação registrada para os casos apresenta melhor qualidade.

Declaração de Nascimento; Confiabilidade e Validade; Registros

\section{Colaboradores}

M. F. Almeida, G. P. Alencar, I. França Jr., H. M. D. Novaes, A. A. F. Siqueira e D. Schoeps participaram da análise dos dados, redação e revisão do texto. O. Campbell e L. C. Rodrigues participaram com sugestões na análise dos resultados. 


\section{Referências}

1. Ministério da Saúde. Indicadores básicos de Saúde. http://www.datasus.gov.br (acessado em 12/ Nov/2004).

2. Silva RI, Theme Filha MM, Noronha CP. Sistema de Informações sobre Nascidos Vivos na Cidade do Rio de Janeiro 1993/1996. Inf Epidemiol SUS 1997; VI:34-48.

3. Carvalho DM. Grandes sistemas nacionais de informação em saúde: revisão e discussão da situação atual. Inf Epidemiol SUS 1997; VI: 35-41.

4. Almeida MF, Alencar GP. Informações em saúde: necessidade de introdução de mecanismos de gerenciamento dos sistemas. Inf Epidemiol SUS 2000; 9:241-59

5. Schramm JMA, Szwarcwald CL. Diferenciais nas taxas de mortalidade neonatal e natimortalidade hospitalares no Brasil: um estudo com base no Sistema de Informações Hospitalares do Sistema Único de Saúde (SIH/SUS). Cad Saúde Pública 2000; 16:1031-40.

6. Kirby RS. The quality of vital perinatal statistics data, with special reference to prenatal care. Paediatr Perinat Epidemiol 1997; 11:122-8.

7. Mello-Jorge MHP, Gotlieb SLD, Soboll MLMS, Almeida MF, Oliveira H. Avaliação do Sistema de Informações sobre Nascidos Vivos: primeira avaliação dos dados brasileiros. Rev Saúde Pública 1993; 27 Suppl:1-45.

8. Piper JM, Mitchell Jr. EF, Snowden M, Hall C, Adams M, Taylor P. Validation of 1989 Tennessee Birth certificates using maternal and newborn hospital records. Am J Epidemiol 1993; 137:75868.
9. Silva AAM, Ribeiro VS, Borba Júnior AF, Coimbra LC, Silva RA. Avaliação da qualidade dos dados do Sistema de Informações sobre nascidos vivos em 1997-1998. Rev Saúde Pública 2001; 35:508-14.

10. McDermott J, Drews C, Green D, Berg C. Evaluation of prenatal care information on birth certificates. Paediatr Perinat Epidemiol 1997; 11:105-21.

11. Theme Filha MM, Gama SGN, Cunha CB, Leal MC. Confiabilidade do sistema de Informações sobre Nascidos Vivos Hospitalares no Município do Rio de Janeiro, 1999-2001. Cad Saúde Pública 2004; 20 Suppl 1:583-91.

12. Reichman NE, Hade EM. Validation of birth certificate data: a study of women in New Jersey's Health Start Program. Ann Epidemiol 2001; 11: 186-93.

13. Clark K, Fu CM, Burnett C. Accuracy of birth certificate data regarding the amount, timing, and adequacy of prenatal care using prenatal clinic medical records as referents. Am J Epidemiol 1997; 145:68-71.

14. Pereira MG. Epidemiologia: teoria e prática. Rio de Janeiro: Guanabara-Koogan; 1999.

15. Watkins ML, Edmonds L, McClearn A, Mullins L, Mulinare J, Khoury M. The surveillance of birth defects: the usefulness of the revised US Standard Birth Certificate. Am J Public Health 1996; 86:731-4.

16. Berquó ES. Fatores estatísticos e dinâmicos. In: Santos JLF, Levy MSF, Szmrecsányi T, organizadores. Dinâmica da população. São Paulo: T. A. Queiroz; 1980. p. 21-85.

17. Buescher PA, Taylor KP, Davis MH, Bowling JM. The quality of the new birth certificate data: a validation study in North Carolina. Am J Public Health 1993; 83:1163-5.

Recebido em 06/Dez/2004

Versão final reapresentada em 18/Ago/2005 Aprovado em 28/Set/2005 